Méndez-Venegas, M., Jiménez, C., Bassey-Fallas, G., \& Cortés, J. (2021). Condición del arrecife coralino de Playa Blanca, Punta Gorda, uno de los arrecifes más extensos de la costa Pacífica de Costa Rica. Revista de Biología Tropical, 69(Suppl. 2), S194-S207. https://doi.org/10.15517/rbt. v69iS2.48742

Biologín Tropical

https://doi.org/10.15517/rbt.v69iS2.48742

\title{
Condición del arrecife coralino de Playa Blanca, Punta Gorda, uno de los arrecifes más extensos de la costa Pacífica de Costa Rica
}

\author{
Mauricio Méndez-Venegas ${ }^{1}$; (D https://orcid.org/0000-0003-4855-3731 \\ Carlos Jiménez ${ }^{2,3}$; (iD https://orcid.org/0000-0003-3413-6662 \\ Giovanni Bassey-Fallas4; (D) https://orcid.org/0000-0002-9900-9069 \\ Jorge Cortés ${ }^{5}$; (D) https://orcid.org/0000-0001-7004-8649
}

1. Ministerio de Ambiente y Energía, Sistema Nacional de Áreas de Conservación, Costa Rica; mauricio.mendez@sinac.go.cr

2. Enalia Physis Environmental Research Centre, Acropoleos 2, Aglantzia 2101, Nicosia, Cyprus; c.jimenez@enaliaphysis.org.cy

3. The Cyprus Institute, Athalassa Campus, P.O. Box 27456, 1645, Nicosia, Cyprus

4. Sede de Guanacaste, Universidad de Costa Rica, Sede Regional de Guanacaste, Costa Rica; bassey33cr@gmail.com

5. Centro de Investigación en Ciencias del Mar y Limnología (CIMAR), Universidad de Costa Rica, 11501-2060 San José, Costa Rica; jorge.cortes@ucr.ac.cr

Recibido 30-I-2021. Corregido 14-IV-2021. Aceptado 20-VIII-2021.

\section{ABSTRACT \\ Condition of the Playa Blanca coral reef, Punta Gorda, one of the most extensive reefs on the Pacific of Costa Rica}

Introduction: Coral reefs are negatively affected by different anthropogenic and environmental causes in the Gulf of Papagayo, Costa Rican Pacific coast.

Objective: The health status of the Playa Blanca coral reef was evaluated.

Methods: Between August 2010 and September 2011, the percentage of live coral cover (LCC) per species and of the macroalgae Caulerpa sertularoides was determined in four sectors and at three depths of the coral reef; the annual growth of Pocillopora damicornis and P. elegans was measured and related to temperature, nutrients, wind, sun irradiation and rain.

Results: 14 species of corals were found, two of them, Fungia curvata and Leptoseris papyracea, only as skeletons. The area with the highest LCC was offshore in the middle zone of Playa Blanca. The species with the highest coverage in all depth ranges was $P$. damicornis, followed by $P$. elegans. The LCC decreased from $29 \%$ in September 2010 to $1 \%$ in October 2010 due to a mass coral mortality caused by harmful algal blooms. The growth rate of $P$. damicornis $\left(26.0 \pm 8.9 \mathrm{~mm}_{\text {year }}{ }^{-1}\right)$ and $P$. elegans $\left(27.9 \pm 4.5 \mathrm{~mm}\right.$ year $\left.^{-1}\right)$ did not show significant differences between depths or location in the reef.

Conclusions: The exuberant Playa Blanca coral reef experienced a sudden drop in LCC similar to those described by Glynn et al. (1983). This suggests that cycles of mortality and natural recovery of coral reefs occur, although anthropogenic pressures reduce the resilience capacity of the reefs. Spatial planning, both on land and in the sea, is vital for adequate human development, in which the increase in the resilience of coral ecosystems is incorporated into the analysis.

Key words: coral reef; Gulf of Papagayo; environmental variables; mass mortality; coral growth. 
Los arrecifes coralinos son afectados negativamente por diferentes causas, como elevadas temperaturas del mar (Jiménez \& Cortés, 2001; Jiménez \& Cortés, 2003a; Romero-Torres et al., 2020), alta irradiación solar (Brown, 1997; Lesser, Stochaj, Tapley, \& Shick, 1990), enfermedades bacterianas (Byers, 2020), Floraciones Algales Nocivas (FAN's) (Alvarado et al., 2018; Guzmán, Cortés, Glynn, \& Richmond, 1990), bajas condiciones de luz, alta turbidez, sedimentación, salinidad anormal, contaminación (Brown, 1997; Cortés \& Reyes-Bonilla, 2017; Suggett \& Smith, 2020; Westmacott, Teleki, Wells, \& West, 2000) y acidificación del océano (Kleypas \& Yates, 2009).

Las floraciones de microalgas, o FAN, son de particular interés. Se han registrado en diferentes partes del mundo episodios de mortalidad de extensos parches coralinos y otros organismos marinos debido a sus toxinas (Arias-Godínez et al., 2021; Bauman, Burt, Feary, Marquis, \& Usseglio, 2010; Stuhldreier, Sánchez-Noguera, Roth, et al., 2015). Estas floraciones involucran la presencia de varias especies de dinoflagelados, diatomeas, cianobacterias, entre otros (Calvo-Vargas, BerrocalArtavia, \& Boza-Abarca, 2016). La entrada de nutrientes al mar proveniente de la escorrentía promueve la ocurrencia de FAN (Kruk et al., 2021). Esto puede modificar la composición de la comunidad béntica a medida que se desplaza a lo largo de la costa y según las condiciones ambientales en que se desarrollan las FAN (Guzmán et al., 1990; Mulholland et al., 2009; Quijano-Scheggia et al., 2012; SierraBeltrán et al., 2004; Vargas, Freer, Guzmán, \& Vargas, 2008).

En el Golfo de Papagayo, Pacífico Norte de Costa Rica, los ambientes coralinos están expuestos a procesos estacionales de afloramiento de aguas frías producto de la acción de los vientos alisios, lo que provoca disminución en la temperatura y oxigeno del agua y mayor acidez y disponibilidad de nutrientes arrastrados desde el fondo marino (Alfaro et al., 2012; Rixen, Jiménez, \& Cortés, 2012; Sánchez-Noguera et al., 2018; Stuhldreier, Sánchez-Noguera, Rixen, et al., 2015; Stuhldreier,
Sánchez-Noguera, Roth, et al., 2015). Sin embargo, los corales se encuentran bien desarrollados y presentan tasas de crecimiento tan altas como las de sitios en donde no se exponen al afloramiento de aguas frías del fondo (Cortés \& Jiménez, 2003).

En el caso del arrecife coralino de Playa Blanca, Punta Gorda, en el Golfo de Papagayo, en 1978 se encontraba prácticamente muerto, con porcentajes de cobertura de coral vivo muy bajas (0-0.1\%), exclusivamente de Psammocora stellata (Glynn, Druffel, \& Dunbar, 1983; Glynn et al., 2017). Así mismo, otros arrecifes en la zona del Golfo de Papagayo presentaron coberturas de coral vivo muy bajas, de alrededor del $1 \%$ y en su mayoría las colonias muertas eran del género Pocillopora (Glynn et al., 1983). Desde entonces, estos arrecifes han experimentado una serie de incrementos y declives en la cobertura de coral vivo (Jiménez, 2001a; Jiménez, 2007; Jiménez, Bassey, Segura, \& Cortés, 2010; Jiménez, Cortés, León, \& Ruíz, 2001), con los consecuentes impactos negativos sobre los organismos arrecifales y actividades asociadas. La zona cercana a Playa Blanca ha tenido un crecimiento turístico acelerado, con los impactos adversos propios de este tipo de actividades (Alvarado et al., 2018).

En esta investigación se determinó la composición de especies de coral del arrecife coralino de Playa Blanca, entre agosto de 2010 y setiembre de 2011, periodo en que ocurrieron cambios significativos en la condición general del arrecife, asociado al comportamiento de variables ambientales y FAN. Adicionalmente, se determinaron las tasas de crecimiento de la especie Pocillopora en función de la profundidad y posición en el arrecife, todo esto con el fin de determinar los sitios propicios para la implementación de acciones de conservación del arrecife.

\section{MATERIALES Y MÉTODOS}

Sitios de muestreo: El arrecife coralino de Playa Blanca (10³2'26.13" N \& 85'46'03.89" O y $10^{\circ} 32^{\prime} 00.10^{\prime \prime} \mathrm{N} \& 85^{\circ} 45^{\prime} 36.29^{\prime \prime}$ O) se extiende paralelo a la costa desde el extremo 
oeste de Playa Matapalo hasta Punta Matapalito. Presenta zonas someras o lagunas con plataformas y canales y áreas con frentes seguidos de un declive que se extiende hasta metros de profundidad (Jiménez, 2007). Para el presente trabajo se establecieron cuatro sitios de muestreo (Fig. 1) con tres estaciones cada uno, en los ámbitos de profundidad menor (2-6 $\mathrm{m}$ de profundidad), media (7-11 $\mathrm{m}$ de profundidad) y mayor (12-17 $\mathrm{m}$ de profundidad), excepto en la estación 4 , donde no existen formaciones coralinas en el ámbito de profundidad mayor.

Variables ambientales: Las variables ambientales analizadas incluyeron temperatura del agua, utilizando un sensor de temperatura (ONSET, HOBO Pro v2 $\pm 0.2{ }^{\circ} \mathrm{C}$, colocado en la estación media del sitio 3; Fig. 1), datos satelitales de temperatura superficial del mar $\left(1^{\circ} \times 1^{\circ}\right.$ cuadrícula centrada en $10.5^{\circ} \mathrm{N} \&$ $86.5^{\circ} \mathrm{O}$ ) del análisis de interpretación óptima Reynolds NCEP (Reynolds \& Smith, 1994) y la salinidad del agua, medida con un refractómetro (EXTECH RF20 resolución de 1\%).
Información de radiación solar $\left(\mathrm{MJ} \mathrm{m}^{-2}\right)$, velocidad del viento $\left(\mathrm{m} \mathrm{s}^{-}\right)$y precipitación anual $(\mathrm{mm})$ se obtuvieron de la estación meteorológica más cercana ubicada en el aeropuerto de Liberia $\left(10^{\circ} 35^{\prime} 36^{\prime \prime} \mathrm{N} \& 85^{\circ} 35^{\prime} 30^{\prime}\right.$ " O, elevación de $54 \mathrm{msnm})$.

Los sedimentos en resuspensión fueron medidos con trampas de PVC (5 cm de diámetro, $25 \mathrm{~cm}$ de largo) colocadas a $50 \mathrm{~cm}$ sobre el fondo y sujetas a varillas de hierro, colocando tres trampas en cada una de las estaciones de muestreo. Las trampas se reemplazaron en cada gira, aproximadamente cada 28 a 30 días. $\mathrm{La}$ tasa de sedimento $\left(\mathrm{mg} \mathrm{cm}^{-2}\right.$ día) se determinó por el peso del sedimento seco. Para determinar nutrimentos (nitrógeno inorgánico disuelto y el fósforo disuelto) y los sedimentos en suspensión (Rogers, Fitz, Gilnack, Beets \& Hardin, 1984), se tomaron 11 muestras mensuales (de marzo 2011 a marzo 2012) del agua superficial ( $1 \mathrm{~m}$ de profundidad) frente a Playa Matapalo (Fig. 1). Se seleccionó este lugar ya que está muy cerca de Playa Blanca y se ubica en la desembocadura de la Quebrada Huesera, que pasa

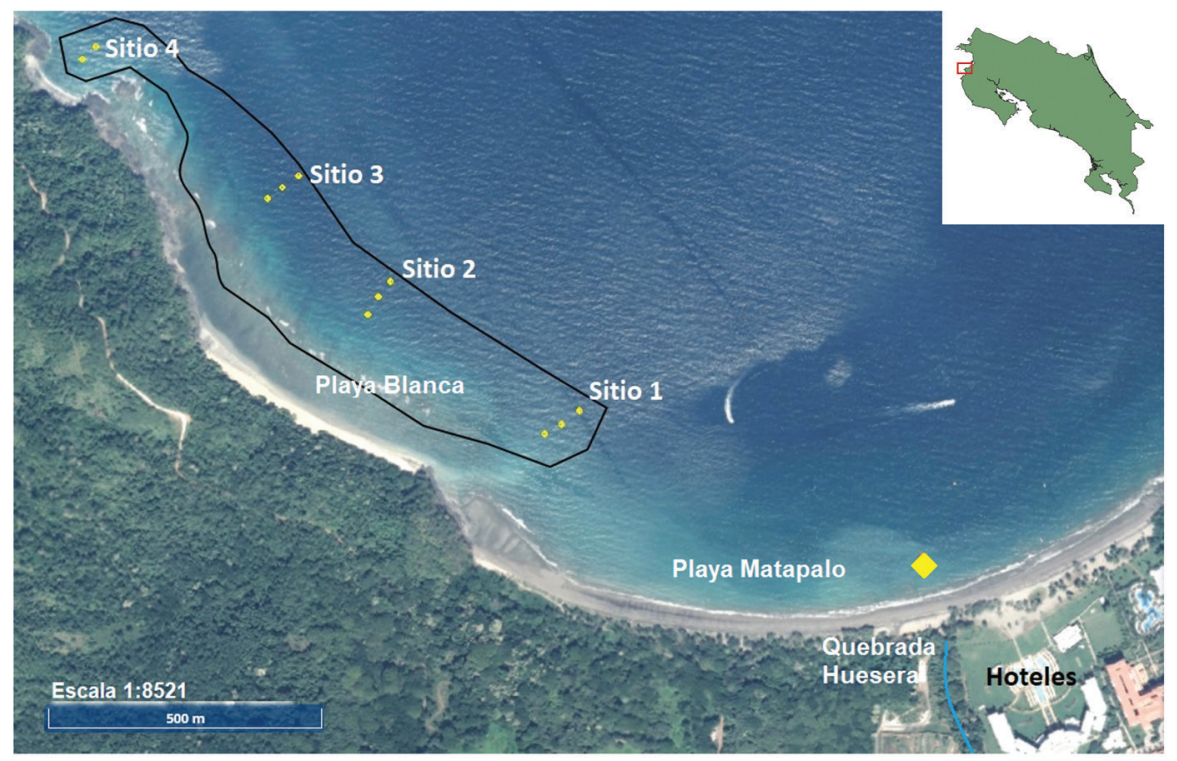

Fig. 1. Ubicación de las once estaciones de muestreo (círculos amarillos) en el arrecife coralino de Playa Blanca, Pacífico Norte de Costa Rica. El punto de muestreo para calidad de agua se indica con un rombo en color amarillo. Escala=1:8521. Fuente: www.snit.go.cr

Fig. 1. Location of the eleven sampling stations (yellow circles) in the Playa Blanca coral reef, North Pacific of Costa Rica. The sampling point for water quality is indicated by a yellow diamond. Scale $=1: 8521$. Source: www.snit.go.cr 
al oeste del área hotelera cerca del arrecife, de manera que permitiera relacionar los cambios en ese ecosistema con el comportamiento de esas variables.

Porcentaje de cobertura: Entre agosto 2010 y setiembre 2011 se determinó el porcentaje de cobertura de coral vivo por especie y de la macroalga Caulerpa sertularoides. Se utilizó el método de transecto lineal paralelo a la costa con cuatro transectos de $10 \mathrm{~m}$ longitud, utilizando una cadena con eslabones de $2.3 \mathrm{~cm}$ de largo (630 eslabones). Este método se utilizó anteriormente en los arrecifes de Papagayo (Jiménez, 2001a; Jiménez, 2007; Jiménez \& Cortés, 2003a). Para estimar la abundancia relativa se utilizaron las categorías: abundante (especies en al menos tres sitios y ámbitos de profundidad); poco abundante (especies en dos sitios o ámbitos de profundidades); común (especies en al menos tres sitios o tres ámbitos de profundidad); escasa (especies en un sitio o ámbito de profundidad); y esqueletos (restos de los corales).

Se midió el crecimiento anual coralino con el método de tinción con alizarina-roja (Lamberts, 1978) para P. damicornis y P. elegans. Las colonias se tiñeron en las tres estaciones de muestreo de los sitios 1 a 3 y en los tres ámbitos de profundidad ya mencionados, con cinco colonias en cada estación desde diciembre 2010 a noviembre 2011. Una vez pasado el año, los corales fueron retirados y se midió el crecimiento desde la marca de alizarina hasta la punta de las tres ramificaciones principales de cada colonia, con un vernier Manostat $\pm 0.025 \mathrm{~mm}$.

Los valores mensuales de cobertura de coral vivo (CCV), crecimiento de colonias y sedimentos en resuspensión fueron comparados por la prueba no paramétrica Kruskal-Wallis utilizando el software InfoStat (Di Rienzo et al., 2011), lo que permitió relacionar las variables con la profundidad y posición en el arrecife.

\section{RESULTADOS}

Variables ambientales: Los datos de temperatura mostraron el comportamiento característico de la zona de Papagayo a lo largo del año (Alfaro et al., 2012; Jiménez, 2001b; Jiménez et al., 2010), con bajas temperaturas $\left(17.3-25^{\circ} \mathrm{C}\right)$ en los meses de diciembre a abril (afloramientos) y temperaturas más elevadas $\left(28-31^{\circ} \mathrm{C}\right)$ durante el resto del año. Además, los datos del sensor y los datos satelitales muestran un comportamiento estacional similar (Fig. 2).

Los datos meteorológicos muestran un incremento en la velocidad del viento entre los meses de diciembre a abril con un máximo de $5.5 \mathrm{~m} \mathrm{~s}^{-1}$ en febrero, mientras que el mínimo se registró en agosto del 2011 con $1.6 \mathrm{~m} \mathrm{~s}^{-1}$ (Fig. 3A). La radiación solar fue superior al promedio histórico a lo largo de todo el periodo

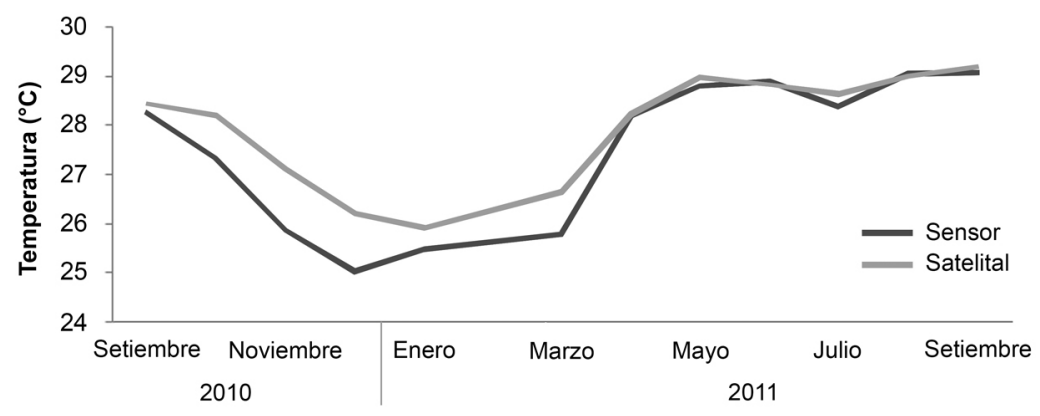

Fig. 2. Comparación del promedio mensual in situ (sensor) y satelital de la temperatura del agua de mar entre 2010 y 2011 en el arrecife coralino de Playa Blanca. Fuente de datos satelitales: http://iridl.ldeo.columbia.edu

Fig. 2. Comparison of the monthly in situ (sensor) and satellite average of seawater temperature between 2010 and 2011 in the Playa Blanca coral reef. Satellite data source: http://iridl.ldeo.columbia.edu 

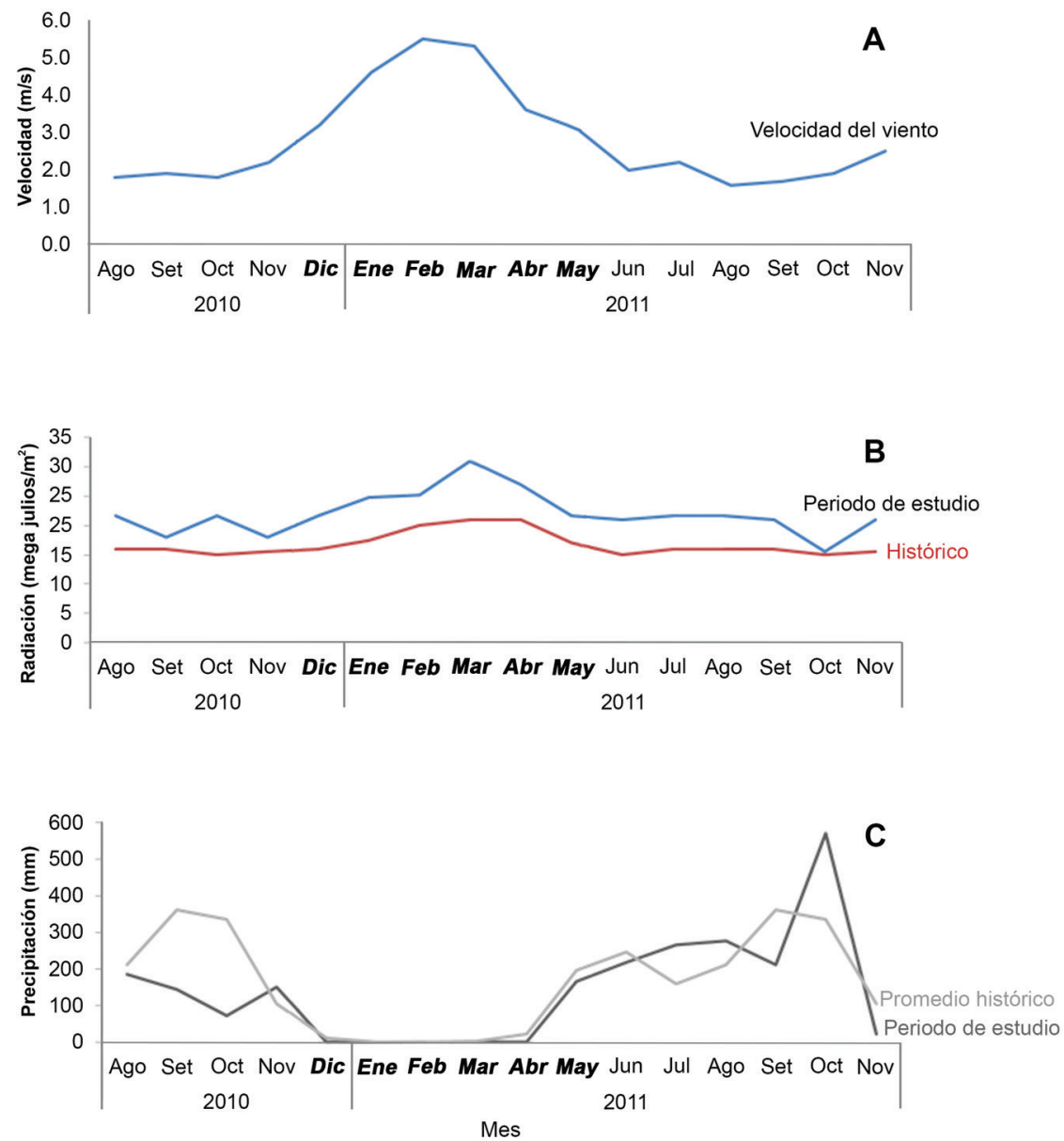

Fig. 3. A) Velocidad del viento $(\mathrm{m} / \mathrm{s})$, B) radiación solar promedio $\left(\mathrm{MJ} / \mathrm{m}^{2}\right)$ y C) precipitación mensual (mm) durante el periodo de estudio en la Estación Meteorológica Liberia, Guanacaste; los meses de afloramiento se muestran en negrita. Fuente: Instituto Metereológico Nacional (2012).

Fig. 3. A) Wind speed $(\mathrm{m} / \mathrm{s})$, B) average solar radiation $\left(\mathrm{MJ} / \mathrm{m}^{2}\right)$ and C) monthly precipitation ( $\left.\mathrm{mm}\right)$ during the study period at the Liberia Meteorological Station, Guanacaste; upwelling season is shown in bold type. Source: National Meteorological Institute (2012).

de estudio con un máximo en marzo de 2011 (31 megajulios $\mathrm{m}^{-2}$ ), excepto en octubre 2011 que fue igual (15 megajulios $\mathrm{m}^{-2}$; Fig. 3B). En octubre de 2010 la precipitación $(73.07 \mathrm{~mm})$ fue menor al promedio histórico $(335.6 \mathrm{~mm})$, mientras que para octubre 2011 la precipitación fue mayor (570.6 mm) a la histórica (Fig. 3C).

La tasa de sedimentos en resuspensión no presentó diferencia significativa a lo largo del año, ni entre sitios $(\mathrm{H}=10.48, \mathrm{P}=0.01)$, ni entre profundidades $(\mathrm{H}=2.30, \mathrm{P}=0.32)$. A pesar de que las diferencias no fueron significativas, el sitio 2 tuvo el promedio más alto de sedimentos en resuspensión $\left(4.2 \pm 0.6 \mathrm{mg} \mathrm{mes}{ }^{-}\right)$, mientras que el ámbito de profundidad media tuvo el promedio mayor $\left(3.6 \pm 0.6 \mathrm{mg} \mathrm{mes}{ }^{-}\right)$.

Con respecto a la variación en las concentraciones estacionales de los nutrimentos, se aprecia un incremento en la cantidad ( $\left.\mathrm{mg} \mathrm{L}^{-}\right)$ de los nutrimentos analizados entre noviembre 2011 y enero 2012. El nitrato para marzo 2011 se encontró muy por encima de los datos 

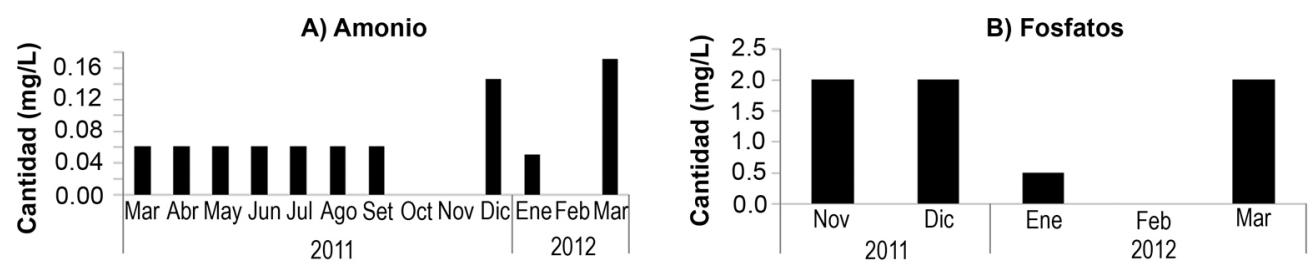

C) Nitratos
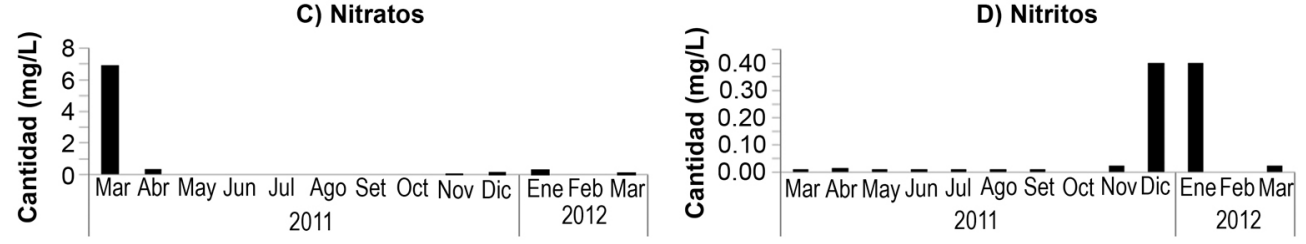

E) Silicio

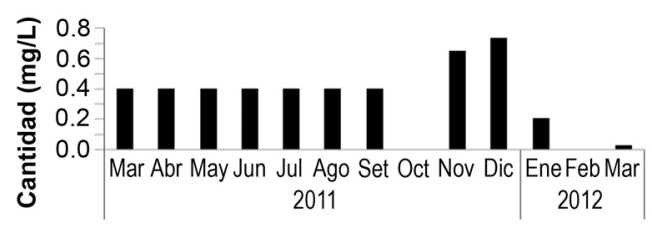

Fig. 4. Nutrimentos analizados por mes en el periodo marzo 2011 a marzo 2012: A) amonio, B) fosfatos, C) nitratos, D) nitritos y E) silicio.

Fig. 4. Nutrients analyzed by month during the period March 2011 to March 2012: A) ammonium, B) phosphates, C) nitrates, D) nitrites and E) silicon.

de los otros meses (6.9 mg L'), así como el fósforo en noviembre y diciembre de 2011 (2 $\left.\mathrm{mg} \mathrm{L}^{-}\right)$(Fig. 4).

Cobertura del fondo: $\mathrm{La} \mathrm{CCV}$ en los cuatro sitios de estudio fue mayor en los meses previos a la llegada de las FAN. En agosto y setiembre 2010 la CCV promedio fue entre 20 $\%$ y $30 \%$ aproximadamente (Fig. 5). En los meses siguientes, octubre 2010 a septiembre 2011 , los valores promedio fueron entre $0 \% \mathrm{y}$ $10 \%$ aproximadamente (Fig. 5).

Para la macroalga $C$. sertularoides se encontró diferencia significativa en el porcentaje promedio de cobertura entre sitios $(0.76 \%$ en el sitio 1 y $25.91 \%$ en el sitio $4, \mathrm{H}=18.56$, $\mathrm{P}$ $=0.003)$, mientras que no se encontró diferencia significativa entre ámbitos de profundidad (3.32\% en profundidad mayor y $15.98 \%$ en profundidad menor, $\mathrm{H}=0.99, \mathrm{P}=0.60$ ), a lo largo del periodo de estudio (Fig. 6).
Se promediaron los valores de $\mathrm{CCV}$ por sitio, con el fin de identificar variaciones durante el período de estudio en el porcentaje de coral vivo según el ámbito de profundidad (Fig. 7). La serie de tiempo resultante de los promedios mensuales muestra una disminución significativa $(\mathrm{P}<0.05)$ después de septiembre 2010 para todos los ámbitos de profundidad.

Pocillopora damicornis estuvo presente en todos los sitios y ámbitos de profundidad (Tabla 1). Dentro de los transectos únicamente se encontraron tres de las 14 especies observadas: P. damicornis (en el $100 \%$ de los transectos), P. elegans (en $82 \%$ ) y P. stellata (en 73 $\%)$. Una especie masiva común en los sitios del arrecife, pero que, sin embargo, no se encontró en ninguno de los transectos fue Pavona clavus. Los corales Pocillopora eydouxi y Porites lobata se hallaron en al menos dos sitios del arrecife, mientras que las demás especies fueron observadas solamente en uno de ellos. 

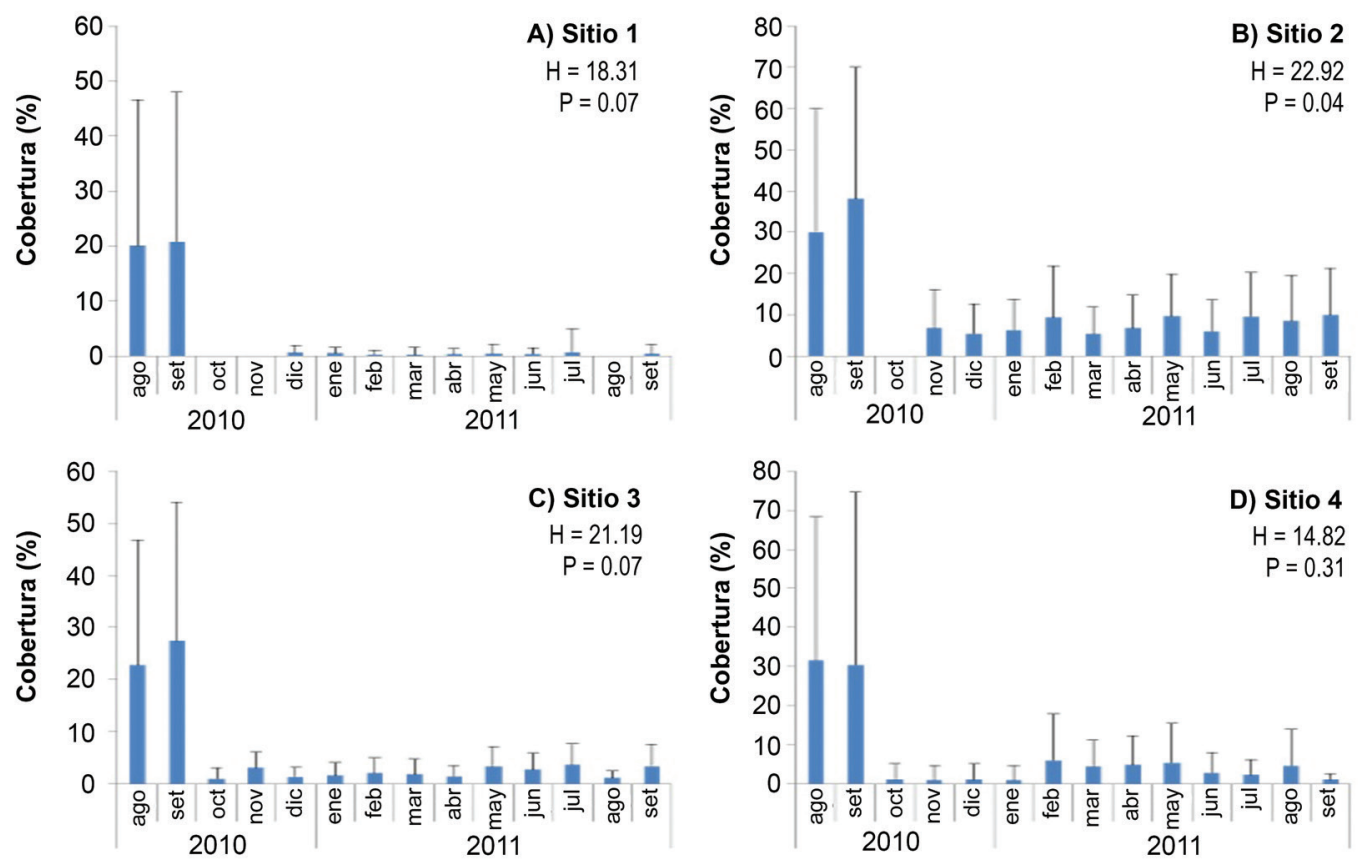

Fig. 5. Porcentaje promedio de cobertura de coral vivo (+ desviación estándar) a lo largo del periodo de estudio para cada uno de los sitios de muestreo ( $\mathrm{N}=12$ transectos en $\operatorname{los}$ sitios 1 a $3, \mathrm{~N}=8$ transectos en el sitio 4). Notar la diferencia en las escalas.

Fig. 5. Average percentage of live coral cover ( + standard deviation) throughout the study period for each of the sampling sites. Number of transects: $\mathrm{N}=12$ transects at sites 1 to $3, \mathrm{~N}=8$ transects at site 4 (note the difference in scales).
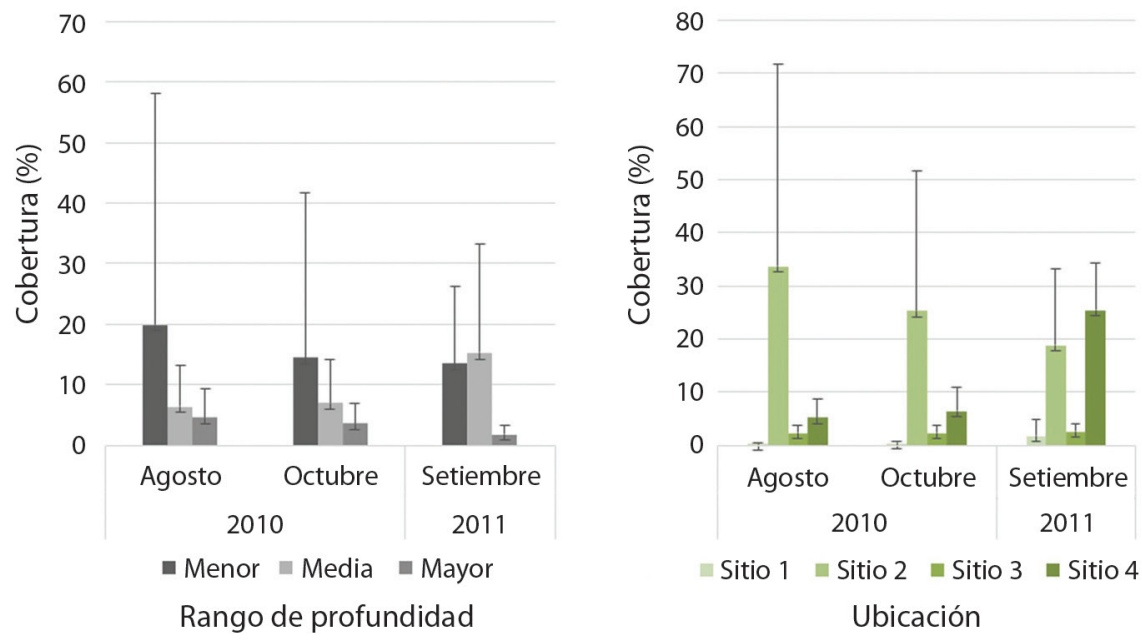

Fig. 6. Porcentaje promedio de Caulerpa sertularoides ( \pm desviación estándar) a lo largo del periodo de estudio en los diferentes rangos de profundidad y para cada uno de los sitios de muestreo $(\mathrm{N}=12$ transectos en $\operatorname{los}$ sitios 1 a $3, \mathrm{~N}=8$ transectos en el sitio 4).

Fig. 6. Average percentage of Caulerpa sertularoides ( \pm standard deviation) throughout the study period in the different depth ranges and for each of the sampling sites $(\mathrm{N}=12$ transects in sites 1 to $3, \mathrm{~N}=8$ transects at site 4$)$. 

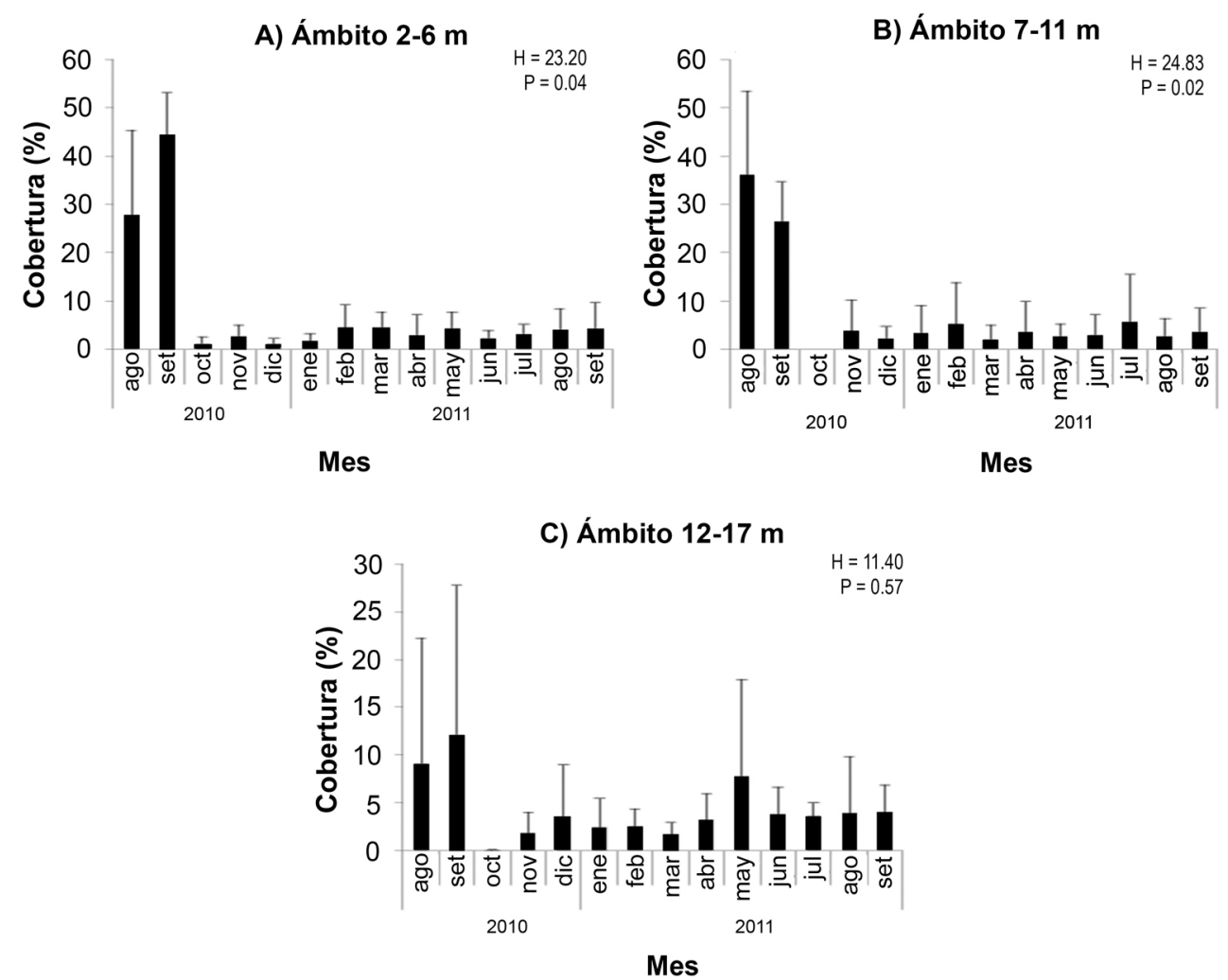

Fig. 7. Porcentaje de cobertura promedio de coral vivo (+ desviación estándar) a lo largo del periodo de estudio por ámbito de profundidad de todos los sitios en conjunto $(\mathrm{N}=12$ transectos en $\operatorname{los}$ sitios 1 a $3, \mathrm{~N}=8$ en el sitio 4). Notar la diferencia de escalas.

Fig. 7. Average live coral cover percentage (+ standard deviation) throughout the study period of all sites combined by depth range $(\mathrm{N}=12$ transects at sites 1 to $3, \mathrm{~N}=8$ at site 4$)$. Note the difference in scales.

Se calculó el promedio mensual de CCV. Como se mencionó anteriormente, hubo una disminución significativa y repentina de la CCV (Fig. 8); disminuyendo de un promedio de entre $26 \%$ y $29 \%$ al comienzo del estudio (agosto y septiembre 2010, respectivamente), este disminuyó a menos del $1 \%$ en octubre 2010. La CCV total durante los meses siguientes fue, en promedio, menos del $4 \%$.

Fig. 8. Porcentaje promedio de cobertura ( \pm desviación estándar) total de coral vivo a lo largo del periodo de estudio en el arrecife coralino de Playa Blanca ( $\mathrm{N}=154$ transectos).

Fig. 8. Average coverage percentage ( \pm standard deviation) of total living coral throughout the study period in the Playa Blanca coral reef $(\mathrm{N}=154$ transects $)$.

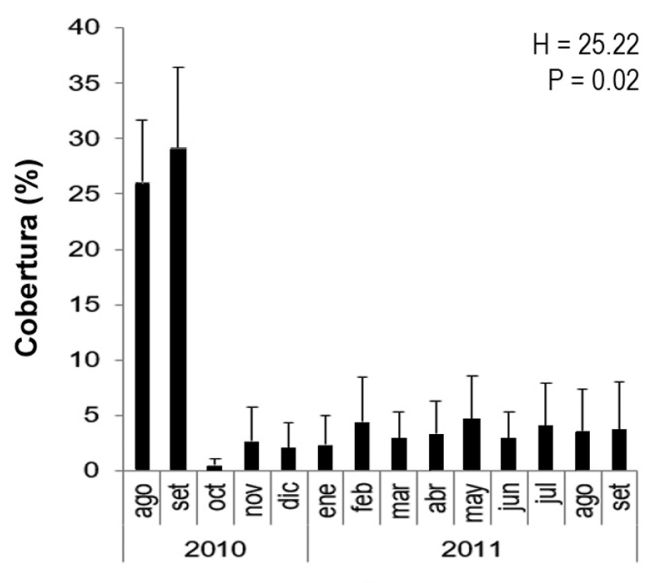

Mes 
TABLA 1

Especies de coral por ámbitos de profundidad y sitios del arrecife de Playa Blanca. Celdas coloreadas indican presencia de colonias vivas, mientras que la $\mathrm{E}$ indica presencia de esqueletos

TABLE 1

Coral species by depth areas and sites of the Playa Blanca reef. Colored cells indicate the presence of living colonies, while the $\mathrm{E}$ indicates the presence of skeletons

\begin{tabular}{|c|c|c|c|c|c|c|c|c|c|c|c|c|}
\hline Profundidad/ámbito & \multicolumn{4}{|c|}{$2-6 \mathrm{~m}$} & \multicolumn{4}{|c|}{$7-11 \mathrm{~m}$} & \multicolumn{3}{|c|}{$12-17 \mathrm{~m}$} & \multirow{2}{*}{ Presencia } \\
\hline Especie / Sitio & 1 & 2 & 3 & 4 & 1 & 2 & 3 & 4 & 1 & 2 & 3 & \\
\hline Pocillopora damicornis & & & & & & & & & & & & Abundante \\
\hline Pocillopora elegans & & & & & & & & & & & & Abundante \\
\hline Pocillopora meandrina & & & & & & & & & & & & Escasa \\
\hline Pocillopora eydouxi & & & & & & & & & & & & Poco abundante \\
\hline Psammocora obtusangula & & & & & & & & & & & & Escasa \\
\hline Psammocora superficialis & & & & & & & & & & & & Escasa \\
\hline Psammocora stellata & & & & & & & & & & & & Abundante \\
\hline Pavona clavus & & & & & & & & & & & & Común \\
\hline Pavona gigantea & & & & & & & & & & & & Escasa \\
\hline Pavona varians & & & & & & & & & & & & Escasa \\
\hline Porites lobata & & & & & & & & & & & & Poco abundante \\
\hline Gardineroseris planulata & & & & & & & & & & & & Escasa \\
\hline Leptoseris papyracea & & & & & & & & & $\mathrm{E}$ & & & Rara \\
\hline Fungia curvata & & & E & & & & & & & & & Rara \\
\hline
\end{tabular}

Crecimiento coralino: El crecimiento anual promedio de $P$. damicornis y $P$. elegans fue similar entre ambas especies $(26.0 \pm 8.9$ $\mathrm{mm}$ año-1 y $27.9 \pm 4.5 \mathrm{~mm}$ año ${ }^{-1}$, respectivamente; $\mathrm{P}>0.05$ ). Dado que el número de colonias teñidas de cada una de las especies fue reducido (10 y 9 colonias, respectivamente) no es posible un adecuado análisis estadístico de los resultados. Sin embargo, ambas especies mostraron diferencia significativa en el crecimiento de las colonias entre sitios $(H=14.87$, $\mathrm{P}<0.001$; y $\mathrm{H}=8.36, \mathrm{P}=0.004$, respectivamente) aunque no por ámbitos de profundidad $(\mathrm{H}=1.12, \mathrm{P}=0.67$; y $\mathrm{H}=0.55, \mathrm{P}=0.87$, respectivamente) (Fig. 9).

\section{DISCUSIÓN}

Las 14 especies coralinas encontradas coinciden con las reportadas por Cortés y Jiménez (2003), aunque la cantidad de especies es menor en el presente estudio. La zona del arrecife con la mayor cobertura fue el sitio 2 (frente a Playa Blanca) y la especie con la mayor cobertura en todos los ámbitos de profundidad fue $P$. damicornis, seguido de $P$. elegans. El sitio 1 presentó la cobertura de coral vivo menor, lo que podría estar relacionado con la cercanía de las quebradas en Playa Matapalo, las cuales aportan sedimentos durante la época lluviosa (seis meses de duración) que afectan negativamente la salud de las colonias coralinas. Esto sugiere la posibilidad de ciclos de mortalidad y recuperación natural de los arrecifes coralinos.

Al inicio de la investigación, el arrecife coralino de Playa Blanca presentaba en general una condición buena, dada la cobertura de coral vivo promedio (26.1\%), aunque con un porcentaje menor que la cobertura descrita por Cortés y Jiménez (2003) para Bahía Culebra (44 \%) y para Playa Guacamaya (40 \%) (Jiménez et al., 2010). Esta condición se mantuvo hasta setiembre 2010, cuando la cobertura de coral vivo descendió significativamente hasta $0.1 \%$ en uno de los sitios. Posterior al evento de mortalidad, el arrecife coralino de Playa Blanca presentó condiciones de cobertura de 

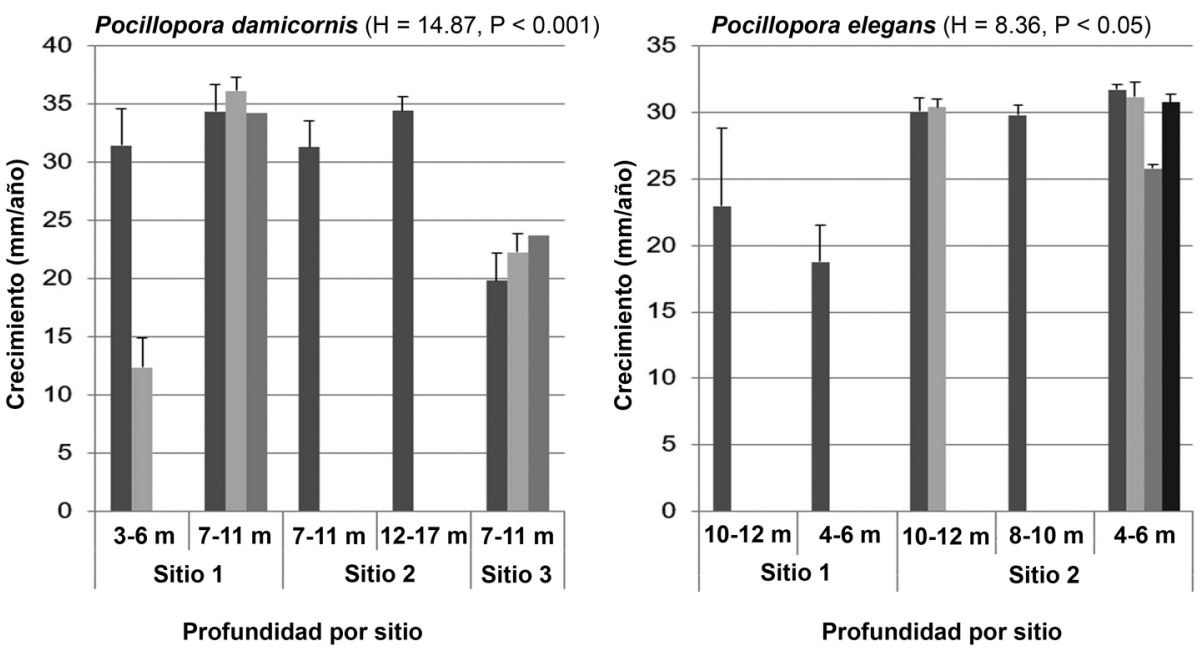

Fig. 9. Promedio de crecimiento ( $\mathrm{mm} /$ año \pm desviación estándar) de $P$. damicornis y P. elegans, por ámbito de profundidad y por sitio de estudio. Cada una de las barras corresponde a una colonia diferente, cada colonia fue medida tres veces.

Fig. 9. Average growth ( $\mathrm{mm} / \mathrm{year} \pm$ standard deviation) of $P$. damicornis and $P$. elegans, by depth range and by study site. Each of the bars corresponds to a different colony, each colony was measured three times.

coral vivo similares a las descritas por Glynn et al. (1983) cuando estudió los arrecifes de esta zona, en su mayoría muertos, con una cobertura máxima del $3 \%$ en 1978.

Durante los meses previos a la mortalidad coralina en el arrecife de Playa Blanca, la temperatura superficial del mar fue en promedio de $28.2 \pm 0.2{ }^{\circ} \mathrm{C}$, con una temperatura máxima de $28.9{ }^{\circ} \mathrm{C}$, lo cual se encuentra dentro de lo usual para la época del año (Alfaro et al. 2012; Jiménez, 2001b; Jiménez et al., 2010). En otros eventos de mortalidad coralina registrados para el área se reporta un promedio de $28.5 \pm 0.7^{\circ} \mathrm{C}$, con un ámbito de entre 26.3 a $30.3{ }^{\circ} \mathrm{C}$ (Jiménez et al., 2001). Reyes-Bonilla, Carriquiry, LeyteMorales y Cupul-Magaña (2002) reportan que los blanqueamientos ocurrieron en la costa oeste de México luego de que la temperatura superficial del mar fuera igual o superior a los $30{ }^{\circ} \mathrm{C}$ por más de 60 días. En otros lugares como las Islas Carolinas, el blanqueamiento y la mortalidad de los corales ocurrió alrededor de $31{ }^{\circ} \mathrm{C}$ (Bruno, Siddon, Witman, Colin, \& Toscano, 2001). Durante la ocurrencia del blanqueamiento la temperatura estuvo por debajo de los $29^{\circ} \mathrm{C}$, lo que sugiere que está no fue la causante de la mortalidad observada.
La condición que parece haber ocasionado blanqueamiento de las colonias fue la presencia de una extensa y prolongada marea roja (FAN) observada en Playa Blanca y Matapalo alrededor de la tercera semana de setiembre 2010 y hasta mediados de octubre. La FAN coincide con la pérdida de coloración y/o tejido de los corales y la mortalidad de parches enteros de colonias de especies del género Pocillopora. Existen registros de episodios de mortalidad de extensos parches coralinos y otros organismos marinos, como consecuencia de la producción de toxinas por parte de dinoflagelados que componen una FAN (Guzmán et al., 1990; Mulholland et al., 2009; Quijano-Scheggia et al., 2012; Sierra-Beltrán et al., 2004, Vargas et al., 2008).

Otros factores que pudieron contribuir a que se diera el evento de mortalidad fue el promedio de radiación solar registrado para el 2010, el cual desde agosto se encontraba por encima del promedio histórico (21.7 MJ $\mathrm{m}^{-2}$ ) (Fig. 3B), lo que pudo contribuir con el incremento en el estrés sobre las colonias coralinas, dada la baja precipitación y velocidad del viento que contribuyeron a una mayor penetración de la luz y con ello el incremento en la 
radiación recibida por las colonias. (Jiménez et al., 2001). En el caso de la precipitación, ésta fue relativamente baja en agosto, respecto del promedio histórico (144 mm), posiblemente, como resultado de El Niño 2009-2010 que estaba terminando y el paso a la fase La Niña (NOAA, 2010). Sin embargo, en setiembre de 2011 se observó un aumento de la precipitación y la escorrentía, lo que produjo un incremento en las cargas de sedimentos que llegan al arrecife (Fig. 3C), aunque no se reflejó de la misma manera en la carga de nutrientes. Lo anterior sugiere que la combinación de factores que en su conjunto generaron condiciones de estrés que contribuyeron a desencadenar el evento de mortalidad de las colonias, frente a una FAN (Alvarado et al., 2018).

Posterior al evento de mortalidad coralina, se observó la recuperación de algunas colonias a partir de pequeñas secciones de tejido vivo. Dos meses después, las colonias que sobrevivieron habían recuperado la mayor parte del tejido (Fig. 8). Por otro lado, este evento no provocó cambios significativos en la cobertura de $C$. sertularoides, debido a que el incremento en su cobertura depende de la disponibilidad de nutrientes (Fernández-García et al., 2012).

Las tasas más altas de resuspensión de sedimentos fueron registradas en enero y febrero 2011, coincidiendo con la más alta turbulencia del agua, velocidad del viento y oleajes. El incremento en las concentraciones de los nutrimentos durante los meses de noviembre a enero podría estar relacionado con el incremento en los vientos alisios propios de la época de afloramiento de Papagayo (Vargas, 2002). El análisis de nutrientes muestra que los fosfatos, nitritos, silicios y amonio tuvieron valores mayores a los obtenidos por Fernández-García et al. (2012), mientras que los nitratos fueron menores. Al respecto, Saravia-Arguedas, Lugioyo, Suárez-Serrano, Guillén-Watson y Sierra (2019) concluyen que la calidad higiénico-sanitaria de esta zona del Golfo de Papagayo es regular, pues los análisis bacteriológicos indican fuentes puntuales de contaminación a lo largo de la costa. Se debe considerar la posibilidad del arrastre de nutrientes desde las zonas verdes de los hoteles, algunos de los cuales utilizan los efluentes de las plantas de tratamiento para regar estas áreas.

Las tasas de crecimiento de $P$. damicornis $\left(23.4 \pm 7.4 \mathrm{~mm}^{-}\right)$y $P$. elegans $\left(24.9 \pm 3.9 \mathrm{~mm}^{-}\right)$ en Playa Blanca fueron menores a las reportadas por Jiménez y Cortés (2003b) $(47.8 \pm 12.4$ y $44.5 \pm 7.6$, respectivamente) y Guzmán y Cortés (1989) (P. elegans $\left.=34.8 \mathrm{~mm}^{-}\right)$en diferentes localidades de Costa Rica. Al comparar el crecimiento de las colonias, según la especie por ámbito de profundidad, la tasa de crecimiento reportada por Guzmán y Cortés (1989) es superior a la de Playa Blanca. Para explicar las menores tasas de crecimiento es necesario considerar los efectos potenciales y altamente probables en las colonias del evento de mortalidad de 2010. A pesar de que las colonias experimentales sobrevivieron a la mortalidad generalizada de los corales en Playa Blanca, los factores ambientales antes mencionados posiblemente no propiciaron las condiciones óptimas para el crecimiento de los corales. Otro aspecto que influyó en una tasa de crecimiento menor a las presentadas en otros sitios es la propuesta por Manzello (2010), quien menciona que, en los últimos 40 años, el crecimiento de $P$. damicornis ha disminuido a una tasa de $0.9 \%$ por año, como consecuencia del incremento en la acidificación del océano (SánchezNoguera et al., 2018). Tanzi, Brown, Tudhope y Dunne (2009) plantean que el incremento en la temperatura superficial del mar ha llevado a que la tasa de calcificación y de crecimiento lineal de Pocillopora lutea haya descendido entre un $19.4 \%$ y $23.4 \%$, respectivamente. Esto podría contribuir a una tasa de crecimiento menor a las registradas en sitios cercanos al arrecife de Playa Blanca en años anteriores.

La condición del arrecife de Playa Blanca se asemeja a la situación de los ecosistemas coralinos a lo largo del mundo, pues la cobertura de coral vivo ha descendido de manera preocupante. Esto debe llevar a la implementación de medidas urgentes de manejo activo de estos ecosistemas, desde actividades básicas como la jardinería de corales que ya ha iniciado en la zona de Golfo de Papagayo y en otros sitios 
de Costa Rica, prestando especial atención al estudio genético de las poblaciones para evitar su erosión genética. La determinación de los ciclos reproductivos de las especies de corales de manera que permita articular medidas de manejo de los eventos de reproducción sexual que son poco conocidos en esta región. El ordenamiento espacial tanto en tierra como en el mar son vitales para un adecuado desarrollo humano, en el que incorpore dentro del análisis, el incremento de la capacidad de recuperación de los ecosistemas coralinos.

Declaración de ética: los autores declaran que todos están de acuerdo con esta publicación y que han hecho aportes que justifican su autoría; que no hay conflicto de interés de ningún tipo; y que han cumplido con todos los requisitos y procedimientos éticos y legales pertinentes. Todas las fuentes de financiamiento se detallan plena y claramente en la sección de agradecimientos. El respectivo documento legal firmado se encuentra en los archivos de la revista.

\section{AGRADECIMIENTOS}

Agradecemos al Centro de Investigación en Ciencias del Mar y Limnología (CIMAR) y la Universidad de Costa Rica, por el apoyo a esta investigación.

\section{RESUMEN}

Introducción: Los arrecifes coralinos son afectados negativamente por diferentes causas antropogénicas y ambientales en el Golfo de Papagayo.

Objetivo: En esta investigación, la condición del arrecife coralino de Playa Blanca fue evaluado.

Métodos: Entre agosto de 2010 y setiembre de 2011 el porcentaje de coral vivo por especie y del alga Caulerpa sertularoides fue determinado; el crecimiento anual de $P$. damicornis y $P$. elegans fue medido y relacionado con variables ambientales.

Resultados: Se encontraron 14 especies de corales, dos de ellas, F. curvata y L. papyracea, únicamente como esqueletos. La zona con la mayor cobertura fue en la zona media de la playa. La especie con mayor CCV en todos los ámbitos de profundidad fue $P$. damicornis, seguido de $P$. elegans. La CCV descendió de $29 \%$ en septiembre 2010 a $1 \%$ en octubre 2010. La mortalidad masiva fue causada por una Floración Algal Nociva (FAN). La tasa de crecimiento de P. damicornis $\left(26.0 \pm 8.9 \mathrm{~mm}\right.$ año $\left.{ }^{-1}\right)$ y de $P$. elegans $(27.9$ $\pm 4.5 \mathrm{~mm}$ año ${ }^{-1}$ ), no mostraron diferencias significativas entre profundidades ni por ubicación en el arrecife.

Conclusiones: El exuberante arrecife de coral de Playa Blanca experimentó una regresión repentina de la CCV similar a las descritas por Glynn et al. (1983). Esto sugiere que ocurren ciclos de mortalidad y recuperación natural de los arrecifes de coral, aunque las presiones antropogénicas reducen la capacidad de resiliencia de estos. El ordenamiento espacial, tanto en tierra como en el mar, es vital para un adecuado desarrollo humano, en el que se incorpore en el análisis el aumento de la capacidad de recuperación de los ecosistemas coralinos.

Palabras clave: arrecife coralino; Golfo de Papagayo; variables ambientales; mortalidad masiva; crecimiento de coral.

\section{REFERENCIAS}

Alfaro, E., Cortés, J., Alvarado, J. J., Jimenez, C., León, A., Sánchez-Noguera, C., Nivia-Ruiz, J., \& Ruiz, E. (2012). Clima y temperatura sub-superficial del mar en Bahía Culebra, Golfo de Papagayo, Costa Rica. Revista de Biología Tropical, 60(Suplemento 2), S159-S171. https://doi.org/10.15517/rbt.v60i2.20000

Alvarado, J. J., Beita-Jiménez, A., Mena, S., FernándezGarcía, C., Cortés, J., Sánchez-Noguera, C., Jiménez, C., \& Guzmán-Mora, A. G. (2018). Cuando la conservación no puede seguir el ritmo del desarrollo: estado de salud de los ecosistemas coralinos del Pacífico Norte de Costa Rica. Revista de Biología Tropical, 66(Suplemento 1), S280-S308. https://doi. org/10.15517/rbt.v66i1.33300

Arias-Godínez, G., Jiménez, C., Gamboa, C. Cortés, J., Espinoza, M., Beita-Jiménez, A., Alvarado, J. J. (2021). The effect of coral reef degradation on the trophic structure of reef fishes from Bahía Culebra, North Pacific coast of Costa Rica. Journal of Coastal Conservation, 25, 8. https://doi.org/10.1007/ s11852-021-00802-x

Bauman, A. G., Burt, J. A., Feary, D. A., Marquis, E., \& Usseglio, P. (2010). Tropical harmful algal blooms: An emerging threat to coral reef communities? Marine Pollution Bulletin, 60(11), 2117-2122. https://doi. org/10.1016/j.marpolbul.2010.08.015

Byers, J. E. (2020). Marine parasites and disease in the era of Global Climate Change. Annual Review of Marine Science, 13(1), 397-420. https://doi.org/10.1146/ annurev-marine-031920-100429

Brown, B. E. (1997). Coral bleaching: causes and consequences. Coral Reefs, 16, 129-138. https://doi. org/10.1007/s003380050249 
Bruno, J. F., Siddon, C. E., Witman, J. D., Colin, P. L., \& Toscano, M. A. (2001). El Niño related coral bleaching in Palau, Western Caroline Islands. Coral Reefs, 20, 127-136. https://doi.org/10.1007/s003380100151

Calvo-Vargas, E., Berrocal-Artavia, K., \& Boza-Abarca, J. (2016). Floraciones algales nocivas durante el periodo 2008-2010 en el Golfo de Nicoya, Costa Rica. Revista Ciencias Marinas y Costeras, 8(1), 129-149. http://dx.doi.org/10.15359/revmar.8-1.9

Cortés, J., \& Jiménez, C. (2003). Corals and coral reefs of the Pacific of Costa Rica: history, research and status. In: J. Cortés (Ed.), Latin American Coral Reefs (pp. 361-385). https://doi.org/10.1016/ B978-044451388-5/50017-5

Cortés, J. \& Reyes-Bonilla, H. (2017). Chapter 20: Human influences on Eastern Tropical Pacific coral communities and coral reefs. In: P. W. Glynn, D. P. Manzello, \& I. C. Enochs (Eds.), Coral Reefs of the Eastern Pacific: Persistence and Loss in a Dynamic Environment. (pp. 549-563). Coral Reefs of the World 8. Dordrecht, The Netherlands: Springer Science+Business Media. https://doi.org/10.1007/978-94-017-7499-4

Di Rienzo, J. A., Casanoves, F., Balzarini, M. G., González, L., Tablada, M., \& Robledo, Y. C. (2011). InfoStat versión 2011. Grupo InfoStat, FCA, Universidad Nacional de Córdoba, Argentina. http://www.infostat. com.ar

Fernández-García, C., Cortés, J., Alvarado J. J., \& NiviaRuiz, J. (2012). Physical factors contributing to the benthic dominance of the alga Caulerpa sertularioides (Caulerpaceae, Chlorophyta) in the upwelling Bahía Culebra, north Pacific of Costa Rica. Revista de Biología Tropical, 60(Suplemento 2), S93-S107.

Glynn, P. W., Alvarado, J. J., Banks, S., Cortés, J., Feingold, J. S., Jiménez, C., ... \& Zapata, F. A. (2017). Eastern Pacific coral reef provinces, coral community structure and composition: An overview. In: P. W. Glynn, D. P. Manzello, \& I. C. Enochs (Eds.), Coral Reefs of the Eastern Tropical Pacific: Persistence and Loss in a Dynamic Environment (pp. 107-176). Coral Reefs of the World, 8. Dordrecht: Springer. https://doi.org/10.1007/978-94-017-7499-4_5

Glynn, P. W., Druffel, E., \& Dunbar, R. (1983). A dead Central American coral reef tract: possible link with the Little Ice Age. Journal of Marine Research, 41, 605637. https://doi.org/10.1357/002224083788519740

Guzmán, H. M., \& Cortés, J. (1989). Growth rates of eight species of scleractinian corals in the eastern Pacific (Costa Rica). Bulletin of Marine Science, 44, 1186-1194.

Guzmán, H. M., Cortés, J., Glynn, P. W., \& Richmond, R. H. (1990). Coral mortality associated with dinoflagellate blooms in the eastern Pacific (Costa Rica and Panama). Marine Ecology Progress Series, 60 299-303.
Jiménez, C. (2001a). Arrecifes y ambientes coralinos de Bahía Culebra, Pacífico de Costa Rica: aspectos biológicos, económico-recreativos y de manejo. Revista de Biología Tropical, 49(Suplemento 2), S215-S231.

Jiménez, C. (2001b). Seawater temperature measured at the surface and at two depths (7 and $14 \mathrm{~m})$ in one coral reef at Culebra Bay, Gulf of Papagayo, Costa Rica. Revista de Biología Tropical, 49(Suplemento 2), S153-S161.

Jiménez, C. (2007) Evaluación ecológica rápida del arrecife coralino de Playa Matapalo (Golfo de Papagayo), uno de los arrecifes más extensos en la costa del Pacifico de Costa Rica. Informe para Conservación Internacional, San José, Costa Rica.

Jiménez, C., \& Cortés, J. (2001). Effects of the 1991-92 El Niño on scleractinian corals of the Costa Rican central Pacific coast. Revista de Biología Tropical, 49(Suplemento 2), S239-S250.

Jiménez, C., \& Cortés, J. (2003a). Coral cover change associated to El Niño, eastern Pacific, Costa Rica, 1992-2001. Marine Ecology, 24, 179-192. https://doi. org/10.1046/j.1439-0485.2003.03814.x

Jiménez, C., \& Cortés, J. (2003b). Growth of seven species of sclaractinian corals in an upwelling environment of the eastern Pacific (Golfo de Papagayo, Costa Rica). Bulletin of Marine Science, 72, 187-198.

Jiménez, C., Bassey, G., Segura, Á., \& Cortés, J. (2010). Characterization of the coral communities and reefs of two previously undescribed locations in the upwelling region of Gulf of Papagayo (Costa Rica). Revista Ciencias Marinas y Costeras, 2, 95-108.

Jiménez, C., Cortés, J., León, A., \& Ruíz, E. (2001). Coral bleaching and mortality associated with the 1997-98 El Niño in an upwelling environment in the eastern Pacific (Gulf of Papagayo, Costa Rica). Bulletin of Marine Science, 69, 151-169.

Kleypas, J. A., \& Yates, K. K. (2009). Coral reefs and ocean acidification. Oceanography, 22(4), 108-117. https://www.jstor.org/stable/24861028

Kruk, C., Martínez, A., Martínez de la Escalera, G., Trinchin, R., Manta, G., Segura, A. M., ... \& Calliari, D. (2020). Rapid freshwater discharge on the coastal ocean as a mean of long distance spreading of an unprecedented toxic cyanobacteria bloom. Science of The Total Environment, 754, 142362. https://doi. org/10.1016/j.scitotenv.2020.142362

Lamberts, A. (1978). Coral growth: alizarin method. In: D. Stoddart, \& R. Johannes (Eds.), Coral reefs: research methods (pp. 523-527). Paris, France: UNESCO.

Lesser M. P., Stochaj, W. R., Tapley, D. W., \& Shick, J. M. (1990). Bleaching in coral reef anthozoans: effects of irradiance, ultraviolet radiation, and temperatura on the activities of protective enzymes against 
active oxygen. Coral Reefs, 8, 225-232. https://doi. org/10.1007/BF00265015

Manzello, D. P. (2010). Coral growth with stress and ocean acidification: lessons from the eastern tropical Pacific. Coral Reefs, 29, 749-758. https://doi. org/10.1007/s00338-010-0623-4

Mulholland, M. R., Morse, R. E., Boneillo, G. E., Bernhardt, P. W., Filippino, K. C., Procise, L. A., ... \& Gobler, C. J. (2009). Understanding causes and impacts of the dinoflagellate, Cochlodinium polykrikoides, blooms in the Chesapeake Bay. Estuaries and Coasts, 32, 734-747. https://doi.org/10.1007/ s12237-009-9169-5

NOAA, National Climatic Data Center. (2010). State of the Climate: Global Analysis for Annual 2010. https:// www.ncdc.noaa.gov/sotc/global/201013

Quijano-Scheggia, S., Olivos-Ortiz, A., Bustillos-Guzmán, J. J., Garcés, E., Gaviño-Rodríguez, J. H., GaliciaPérez, M. A., ... \& López-Cortés, D. J. (2012). Bloom of Gymnodinium catenatum in Bahía Santiago and Bahía Manzanillo, Colima, México. Revista de Biología Tropical, 60(1), 173-186.

Reyes-Bonilla, H., Carriquiry, J. D., Leyte-Morales, G. E., \& Cupul-Magaña, A. L. (2002). Effects of the El Niño-Southern Oscillation and the anti-El Niño event (1997-1999) on coral reefs of the western coast of México. Coral Reefs, 21, 368-372. https://doi. org/10.1007/s00338-002-0255-4

Reynolds, R., \& Smith, T. (1994). Improved global sea surface temperature analysis using optimum interpolation. Journal of Climate, 7, 929-948. https://doi. org/10.1175/1520-0442(1994)007<0929:IGSSTA >2 $.0 . \mathrm{CO} ; 2$

Rixen, T., Jimenez, C., \& Cortés, J. (2012). Impact of upwelling events on the sea water carbonate chemistry and dissolved oxygen concentration in the Gulf of Papagayo (Culebra Bay), Costa Rica: Implications for coral reefs. Revista de Biología Tropical, 60(Suplemento 2), S187-S195.

Rogers, C. S., Fitz, H. C., Gilnack, M., Beets, J., \& Hardin, J. (1984). Scleractinian coral recruitment patterns at Salt River submarine canyon, St. Croix, U.S. Virgin Islands. Coral Reefs, 3, 69-76. https://doi. org/10.1007/BF00263756

Romero-Torres, M., Acosta, A., Palacio-Castro, A. M., Treml, E. A., Zapata, F., Paz-García, D. A., \& Porter, J. A. (2020). Coral reef resilience to thermal stress in the Eastern Tropical. Global Change Biology, 26(7), 3880-3890. https://doi.org/10.1111/gcb.15126

Sánchez-Noguera, C., Stuhldreier, I., Cortés, J., Jiménez, C., Morales, Á., Wild, C., \& Rixen, T. (2018). Natural ocean acidification at Papagayo upwelling system (north Pacific Costa Rica): implications for reef development. Biogeosciences, 15(8), 2349-2360. https:// doi.org/10.5194/bg-15-2349-2018

Saravia-Arguedas, A. Y., Lugioyo, G. M., Suárez-Serrano, A., Guillén-Watson, A., \& Sierra, L. (2019). Fuentes terrestres de contaminación que impactan la zona marino-costera del Golfo de Papagayo, Costa Rica. Revista Ciencias Marinas y Costeras, 11(2), 69-84. http://dx.doi.org/10.15359/revmar.11-2.4

Sierra-Beltrán, A. P., Lluch-Cota, D. B., Lluch-Cota, S. E., Cortés-Altamirano, R., Cortés-Lara, M. C., CastilloChávez, M., Carrillo, L., Pacas, L., Víquez, R., \& García-Hansen, I. (2004). Dinámica espacio-temporal de organismos precursores de marea roja en la costa Pacífica de América del Norte y Centroamérica. Revista de Biología Tropical, 52(Suplemento 1), S99-S107.

Stuhldreier, I., Sánchez-Noguera, C., Rixen T., Cortés, J., Morales, A., \& Wild, C. (2015). Effects of seasonal upwelling on inorganic and organic matter dynamics in the water column of eastern Pacific coral reefs. PLoS ONE, 10(11), 1-16. https://doi.org/10.1371/ journal.pone.0142681

Stuhldreier, I., Sánchez-Noguera, C., Roth, F., Cortés, J., Rixen, T., \& Wild, C. (2015). Upwelling increases net primary production of corals and reef-wide gross primary production along the Pacific coast of Costa Rica. Frontiers in Marine Science, 2, 113. https://doi. org/10.3389/fmars.2015.00113

Suggett, D. J., \& Smith, D. J. (2020). Coral bleaching patterns are the outcome of complex biological and environmental networking. Global Change Biology, 26, 68-79. https://doi.org/10.1111/gcb.14871

Tanzi, J. T. I., Brown, B. E., Tudhope, A. W., \& Dunne, R. P. (2009). Decline in skeletal growth of the coral Porites lutea from the Andaman Sea, South Thailand between 1984 and 2005. Coral Reefs, 28, 519-528. https://doi.org/10.1007/s00338-008-0457-5

Vargas, J. M. (2002). Interacción océano-atmósfera: surgencia y generación de anillos en la región de Papagayo. Revista Geográfica de América Central, 1(40), 133-144.

Vargas, M., Freer, E., Guzmán, J. C., \& Vargas, J. C. (2008). Florecimientos de dinoflagelados nocivos en la costa Pacífica de Costa Rica. Hidrobiológica, 18(Suplemento 1), 15-23.

Westmacott, S., Teleki, K., Wells, S., \& West, J. M. (2000). Manejo de arrecifes de coral blanqueados o severamente dañados. Gland, Suiza y Cambridge, Reino Unido: UICN. 